\title{
Promesses, promesses
}

\author{
Richard C. Lewontin
}

Un récent article du New York Times rapporte, qu'à sa demande, les deux sœurs d'une femme lui ont arraché les yeux. Cette femme souffrait d'une maladie chronique, dont la cause, à en croire le prêtre de sa religion vaudou, était qu'un esprit malin avait pris possession de son corps et que la seule solution pour l'exorciser était de lui extirper les yeux. Une croyance populaire très répandue, également partagée par les européens jusqu'à la fin du XVIII" siècle, est que la maladie et la mort proviennent d'états anormaux et exceptionnels, de démons physiologiques sinon spirituels, que l'on doit localiser et exorciser. Selon les archives consultées par Manzoni pour rédiger son I Pressi Sposi, il était couramment admis que la peste milanaise de 1620 avait été propagée par un onguent magique administré par des personnes diaboliques.

Les sociétés technologiquement avancées n'acceptent plus que les esprits du mal soient considérés comme une explication pertinente de la mort et de la maladie, mais le modèle sous-jacent persiste, qui considère que la vie "normale" est une vie en bonne santé, sans souci réel de la mort, par opposition à la vie maladive considérée comme "anormale». Nous pourrons prévenir ou guérir la maladie et repousser la mort dans un futur lointain, éloigné de nos pensées, seulement si nous pouvons trouver les causes individuelles de nos maux. La théorie infectieuse des maladies a été la première étape vers la localisation de l'ennemi et il est universellement les champignons étant maintenant connus, les gens vivent en meilleure santé et plus longtemps. On peut, cependant, émettre quelques doutes sur cette belle histoire. Le taux de mortalité imputable à la tuberculose, l'affection la plus meurtrière en Europe a u XIX ${ }^{e}$ siècle, a montré une décroissance continue et linéaire au cours du temps, et sa pente n'a pas été le moins du monde affectée par la théorie infectieuse de Koch et ses développements ultérieurs, jusqu'à ce que cette maladie cesse d'être une cause significative de décès, et que le coup de grâce lui soit asséné au cours de ce siècle par la chimiothérapie. L'histoire de la plupart des maladies infectieuses mortelles du siècle dernier est à peu près semblable. La seule exception notable a été le taux de mortalité imputable à la variole, maladie pour laquelle le vaccin a été introduit avant la naissance de Koch! Certes, l'espérance de vie des Européens s'est considérablement accrue au cours des cent dernières années, mais cet accroissement est surtout le résultat d'une baisse de la mortalité périnatale et infantile, alors que l'espérance de vie des individus mâles de race blanche de 65 ans ne s'est accrue que de deux ans. Il serait pervers d'affirmer que notre compréhension de l'infection n'a eu que peu d'effet sur la santé et la maladie, mais il convient de rester prudent sur ce que l'on avance à ce propos.

Les microbes, ces démons agents de maladie et de mort, ne sont plus remis en question dans les pays technologiquement avancés. La dernière tentative pour attribuer aux germes les causes de notre mortalité a été le vif intérêt pour l'oncologie virale, mais il n'a pas eu de suite lorsqu'il est apparu que, de toute évidence, les virus ne pouvaient être tenus pour responsables de la plupart des cancers. Nous maîtrisons les germes et leur menace, à quelques exceptions près comme le SIDA, et cependant chacun tombe malade, voire très malade, et l'on continue à mourir sans que la durée de vie s'accroisse notablement. Nous sommes ainsi toujours à la poursuite de nouveaux démons à exorciser dans l'espoir d'accéder enfin à la bonne santé éternelle et à une vie plus longue, même si nul ne croit à l'immortalité physique d'où la souffrance serait bannie. Les microbes étaient la cause de tous nos maux, mais nous avons changé tout cela. Nous avons trouvé les gènes.

Il n'est pas de semaine que les généticiens moléculaires n'annoncent dans la presse la localisation d'un gène nouveau responsable de telle ou telle maladie. Une découverte des plus récentes est celle du "gène du cancer du sein". Il y a là une contradiction troublante. Les biologistes insistent soigneusement sur le fait que la plupart des cancers du sein ne sont pas associés à ce fragment d'ADN et que connaître la localisation d'un gène dont les mutations prédisposent son porteur à une forme de cancer n'apporte aucune piste pour son traitement. Et pourtant, ces mêmes biologistes étaient en rude compétition avec des collègues, dès lors fort dépités, pour localiser le gène. Lorsqu'on interroge les généticiens moléculaires sur la nécessité de localiser et de séquencer un gène 
dont les mutations prédisposent à une maladie, leur réponse adopte une rhétorique révélatrice. Ils parlent de "première étape d'une longue route", de découverte qui "pourrait éventuellement " conduire à un "possible " traitement du dérèglement dans un futur "dont on ne peut préciser l'échéance ».

Pourquoi, si la localisation d'un gène n'est que «la première étape d'une longue route qui pourrait éventuellement conduire à un possible " traitement, la découverte de ce gène se fait-elle au prix de tels investissements financiers et confère-t-elle un tel prestige à ceux qui l'ont faite? Une des raisons pourrait être que les succès antérieurs dans des cas similaires constituent un modèle de ce qu'il y a lieu de faire pour trouver un traitement dans le cas particulier. Mais cet argument ne tient pas : bien que nous connaissions l'existence de centaines de gènes impliqués dans des maladies humaines, ainsi que la séquence de nombre d'entre eux, il n'est pas (encore) d'exemple de développement d'un traitement fondé sur cette connaissance. Dans les cas où des dérèglements génétiques, comme ceux du syndrome de Wilson, de la galactosémie ou de la phénylcétonurie, font l'objet d'un traitement approprié, celui-ci est purement fondé sur des connaissances biochimiques et sur celle du métabolisme intermédiaire de la voie biochimique en question. La séquence d'ADN n'a (encore) absolument rien apporté au traitement. Bien sûr, cela ne signifie pas que l'on ne parviendra jamais à une thérapie génique, et l'on peut spéculer sur les possibilités offertes. Supposons par exemple qu'une mutation se produise dans un $\mathrm{ADN}$, cible d'une protéine qui s'y associe, et qu'elle entraîne une association plus faible et plus incertaine. Il serait alors possible de préparer un peptide qui s'associerait correctement à l'ADN et que l'on pourrait fournir de façon exogène. Mais aucun cas favorable de la sorte ne s'est présenté jusqu'ici.

Autre possibilité: la connaissance de la séquence de l'ADN d'un gène pourrait nous apporter celle d'une protéine inconnue, ou bien encore ses variants pourraient nous renseigner sur les types de défauts qui existent dans une protéine donnée. L'observation d'une séquence d'ADN pourrait ainsi nous informer sur le défaut biochimique. Notons, cependant, deux problèmes liés à cette approche; le premier est qu'il est maintenant clair que les défauts génétiques les plus répandus sont génétiquement hétérogènes. Plus de 300 substitutions de nucléotides dans les gènes codant pour le Facteur VIII et le Facteur IX sont connues, chacune pouvant conduire à l'hémophilie. La thalassémie et la mucoviscidose sont tout aussi hétérogènes. Beaucoup de mutations se sont produites indépendamment dans l'espèce et il existe de nombreuses façons de perturber la transcription et l'épissage d'un gène donné. Le second problème est qu'il n'y a pas de séquence standard représentative de la forme " normale" d'un gène. L'étude des populations a montré que les hommes sont hétérozygotes pour à peu près $0,05 \%$ de leur $\mathrm{ADN}$; il en résulte que deux génomes normaux diffèrent par plus d'un million de nucléotides. Comment saurons-nous, en examinant la séquence d'un individu, s'il a une séquence "normale" du gène?

Quand on les interroge, les généticiens moléculaires admettent que la thérapeutique, au sens usuel du terme, n'est pas le but de l'étude génétique des maladies. C'est plutôt la thérapie génique qui est envisagée. Les techniques pour implanter un ADN donneur dans une population cellulaire suffisamment grande pour que l'on obtienne quelque effet physiologique existent certainement, et elles ne peuvent aller qu'en s'améliorant. En outre, le traitement diététique, relativement peu sophistiqué, des maladies génétiques du métabolisme telles que la phénylcétonurie et la galactosémie a été beaucoup moins couronné de succès que la presse populaire ne l'a prétendu, parce que l'absence des enzymes intracellulaires qui interviennent dans des voies métaboliques complexes ne saurait être simplement compensée par la privation d'un substrat. La thérapie génique offre, en un sens, une possibilité plus fondamentale et plus complète de traitement. Cependant, une fois encore, un optimisme exagéré pourrait conduire à des promesses qui ne peuvent être tenues. A l'heure actuelle, en dépit de nombreuses affirmations, il n'y a pas eu à proprement parler de guérison. Récemment, une forme héréditaire d'hypercholestérolémie a été traitée en recolonisant le foie d'un patient par des cellules génétiquement transformées. Une presse très favorable s'est faite l'écho de la chute importante de la concentration de cholestérol du patient, mais nul ne semble avoir souligné que, même abaissée, cette concentration était encore très sévèrement pathologique.

Le problème, avec la "génomanie" qui pollue maintenant notre vision des maladies et de la mort chez l'homme, est qu'elle confond les causes et les agents. Lorsque les Européens ont cessé de mourir d'infections et de malnutrition, ils ont commencé à mourir de maladies cardiaques, de cancer et d'accidents vasculaires cérébraux. Il existe nécessairement une corrélation négative entre les diverses "causes" de la mort. Puisque chacun doit mourir, ceux qui ne meurent pas de cancer mourront d'une crise cardiaque. De plus, si la théorie mutationniste en vigueur pour la cancérogenèse est la bonne, une thérapie génique qui remplacerait un gène mutant prédisposant à une forme génétique de cancer du sein serait mise en échec par des mutations somatiques du même oncogène ou d'autres. Bien sûr, on pourrait promettre une baisse générale des taux de mutation, mais alors le cœur, ou le foie ou les reins flancheraient. Le cancer, les maladies cardiaques, les attaques ne sont pas les causes de la mort, mais ses agents. L'organisme est une machine et, comme toute machine "normale", l'un des aspects de sa normalité est que ses pièces s'usent les unes après les autres. Bien que je sois prêt à tout moment à remplacer toute pièce défectueuse de ma voiture, le jour viendra où je devrai l'envoyer à la casse. Une histoire populaire du Vermont raconte qu'un homme avait 
une hache qui était dans sa famille depuis cent cinquante ans. Quand on s'émerveillait du soin qui devait avoir été pris de cette hache, il répondait : "Oh oui, on a remplacé le manche quatre fois et le fer deux fois."

La multiplicité des sources génétiques de mort et de maladie est à l'origine de sérieux problèmes éthiques, mais pas de ceux que l'on croit en général. Les aspects éthiques qui émergent habituellement des diagnostics génétiques et de la thérapie génique sont, en fait, davantage une question de jugement que de morale et, lorsque les vrais problèmes éthiques se posent, ce n'est pas leur spécificité génétique qui les fait naître. Aux Etats-Unis, par exemple, les employeurs ont le droit d'enquêter sur les travailleurs qu'ils veulent embaucher et de s'opposer à l'engagement de ceux qui seraient sensibles aux risques du métier. Ce tri leur permet de limiter le coût des assurances de santé et déjoue par avance les pressions auxquelles ils pourraient être soumis pour changer les conditions de travail des ouvriers, tout cela au prix du chômage de quelques-uns. Il ne s'agit pas là, toutefois, d'un problème d'éthique mais d'un problème d'économie politique. Les employeurs ont toujours usé de leur pouvoir et des moyens de coercition dont ils disposent pour s'assurer du contrat le plus favorable possible concernant les salaires et les conditions de travail, tandis que, de leur côté, les travailleurs utilisent tous les moyens en leur possession pour résister au patronat et faire tourner le combat en leur faveur. Bien entendu, les travailleurs pourraient, dans leur propre intérêt, s'opposer au contrôle génétique et réclamer les conditions de travail les plus saines possibles mais, généralement, ils manquent de puissance pour le faire. De fait, à de rares exceptions près, c'est l'employeur qui est en position de force dans le marché du travail et qui tire le meilleur parti du contrat. On pourrait, bien sûr, se demander si le marché du travail capitaliste ne serait pas fondamentalement injuste, mais cela est une autre histoire. est de savoir s'il faut apprendre à une personne, homme ou femme, qu'elle est porteuse d'une mutation qui a une probabilité élevée de s'exprimer et de provoquer une grave maladie. Doit-on, par exemple, "apprendre" à une femme qu'elle est porteuse d'une mutation du "gène du cancer du sein"? Mais, ainsi posée de façon impersonnelle, la question escamote le vrai problème. Qui doit le lui dire, et quelles circonstances ont été à l'origine de l'analyse de ses gènes ? En pratique, les femmes examinées pour ce gène ont un antécédent familial de cancer du sein et sont médicalement suivies à leur propre demande. En tant qu'adultes ayant spécifiquement réclamé un test sur leur propre corps, elles ont le droit absolu d'en connaître le résultat. Qu'il ait été ou non judicieux d'en faire la demande initiale relève de leur propre jugement. Il n'y a pas de conflit entre les principes moraux. $\mathrm{Si}$, en revanche, un enfant est examiné pour effectuer la prédiction génétique d'une possible maladie, il appartient aux parents de décider s'ils doivent ou non lui révéler les résultats de cet examen: c'est aussi une question de jugement et, en dernière analyse, la décision sera prise dans le seul intérêt de l'enfant. Pour les parents, le seul impératif moral de cette décision est l'intérêt de l'enfant tel qu'ils le perçoivent.

Enfin, on affirme souvent que la thérapie génique, particulièrement celle qui pourrait toucher à la lignée germinale, pose des problèmes particuliers d'éthique du fait que les décisions concernent des vies encore non advenues, et que leur impact porte non pas sur ceux qui décident mais, dans un futur plus ou moins éloigné, sur leurs descendants. Cependant, ce n'est qu'un embarras mineur dans l'état actuel de la biologie humaine et de la vie sociale. La décision la plus grave concernant la vie des générations futures, que nous prenons, bien sûr, sans les consulter, est en premier lieu de les concevoir. J'ai connu des cas non isolés d'Européens qui, ayant souffert au cours des années 1930 et 1940 , ont refusé d'avoir des enfants car ils ne voulaient pas qu'ils "naissent dans un tel monde ". Les enfants ne demandent pas à venir, on le leur impose. Et ces conditions sont largement imposées par des décisions personnelles, sociales, et politiques auxquelles ils ne sauraient participer. Rejeter la thérapie génique parce qu'elle affecte les vies à venir revient à prendre grand soin de passer ce que l'on boit, de peur d'avaler un moucheron, et à avaler un chameau (Matt. XXIII, 24, NDT).

Les vrais problèmes éthiques soulevés par notre génomanie sont engendrés par la vision erronée, continuellement renforcée, que nous avons de la maladie et de la mort de l'homme, et de l'affirmation que la génétique détient les réponses à nos problèmes physiques et sociaux les plus aigus. Des généticiens bâtissent leur carrière, deviennent performants et reconnus, grâce à des promesses faites à un public désespéré. Les aspects financiers sont aussi non négligeables. Aux Etats-Unis, tout généticien moléculaire de quelque notoriété possède des intérêts financiers dans une firme biotechnologique, en tant que conseiller scientifique en chef, fondateur, partenaire ou même propriétaire. Les généticiens moléculaires ont bien ouvré pour leur cause en affirmant qu'ils avaient les moyens d'exorciser les démons génétiques qui nous possèdent.

Plus fondamentales encore que cette corruption sont les conséquences morales d'une focalisation sur les anomalies génétiques en tant que source majeure des maladies. Dès lors qu'une condition est considérée comme normale pour la santé et une durée de vie indéfinie et s'oppose à une condition anormale, les êtres humains vont se trouver partagés entre normaux et anormaux, bien et mal tournés, sains et malades. S'il est de la plus haute importance de ramener l'anormal au normal, qu'allonsnous faire si la transformation curative s'avère impossible ? Les vies "anormales" ne vaudraient-elles pas la peine d'être vécues? A en juger au travers des preuves maintes fois apportées au cours de l'Histoire, les malades incurables n'ont pas droit à la vie?

Le modèle qui consiste à considérer comme anormal celui qui est physi- 
quement indésirable nous conduit inéluctablement à l'idée que ceux qui sont socialement indésirables sont aussi anormaux, et que les causes en sont les mêmes. On dit bien qu'il y a des gènes de l'alcoolisme, de la dépendance envers la drogue, de la pulsion criminelle et de la pauvreté. Mais, si les causes des anomalies individuelles et sociales sont les mêmes, elles relèvent des mêmes traitements. Et si ils ne marchent pas, qu'est-ce qui va marcher ? Le danger de la manie qui consiste à interpréter en termes de biologie moléculaire des maladies individuelles et des problèmes sociaux est que la génétique moléculaire ne peut pas offrir, en tous cas pas beaucoup mieux que la théorie infectieuse des maladies, de possibilité d'éradication de la douleur, de la souffrance et de la mort. Elle peut, tout au mieux, nous permettre d'échanger un type de cancer contre un autre. Le problème n'est pas que des centaines de millions soient dépensés pour le projet "génome humain". Ce n'est pas le projet lui-même qui est en cause, mais la symptomatique d'une explication unique des désordres, des maladies et de la mort. Le vrai problème est que, comme conséquence d'une vaste promesse qui ne peut être tenue, il va en résulter un grand cynisme à l'encontre de la science, et finalement, de la raison. Les gens vont-ils alors en revenir à extirper les yeux?

\section{JEAN TAVLITZKI}

Jean Tavlitzki vient de nous quitter à l'âge de 74 ans.

Il commence, en 1949, sa carrière scientifique de généticien à l'Institut de Biologie Physico-Chimique, dans le domaine de la génétique de la levure, sous la direction du Pr. Boris Ephrussi. Ce travail débouche, en 1958, sur sa thèse de Doctorat ès Sciences intitulée "Recherche génétique et physiologique sur le métabolisme de la Co-carboxylase chez des souches de Saccharomyces cerevisiae exigeant la thiamine ".

Il rejoint ensuite le groupe du Pr. Marianne Manago. En 1960, il quitte son poste de chercheur au CNRS pour occuper les fonctions de prof esseur de génétique à l'Université de Paris ; il y montera un laboratoire de recherche à ce qui deviendra plus tard l'Institut Jacques-Monod. Il étudie alors la différenciation cellulaire du champignon du genre Ustilago cynodontis du chiendent.

C'est à partir de cette époque qu'il se penche sur la didactique de l'enseignement de la biologie et de la génétique dans notre pays. Aussi, à partir de 1982, exerce-t-il une haute responsabilité dans les commissions d'enseignement au Ministère de l'Enseignement Supérieur et occupe-t-il la fonction de conseiller scientifique à la Cité des Sciences et de l'Industrie de la Villette.

L'aboutissement en est : la réforme de l'enseignement de la biologie au lycée, la réalisation d'un merveilleux film didactique "le Code génétique", une bande dessinée "la vie c'est féérique ou La formule magique » éditée en 1984, et de nombreuses cassettes sur douze clefs pour la Biologie en 1985.

Jean Tavlitzki a initié, il y a déjà un quart de siècle, les "Rencontres de Méribel" où de jeunes et de moins jeunes chercheurs et enseignants discutent, d'une manière pluridisciplinaire, des concepts et idées nouvelles dans le domaine de la biologie d'aujourd'hui.

Sa passion pour l'art et la manière d'enseigner la biologie et la génétique, en particulier, a été communiquée à tous ses élèves universitaires qui ont essaimé dans l'enseignement et la recherche. Ainsi, sa mémoire, son message continueront-ils à guider de nombreuses générations d'enseignants et de chercheurs en France.

Animé par sa passion d'enseignant, Jean Tavlitzki était aussi un gentilhomme, amoureux de l'art, de la culture, de la musique pour flûte traversière.

Marc Fellous

\section{R.L. Lewontin}

Professeur à l'Université Harvard, Museum of comparative zoology, Harvard University, 26 Oxford Street, Cambridge, MA 02138, États-Unis. 